\title{
The Fall 2004 SDSS Supernova Survey
}

\author{
Masao Sako (KIPAC/Stanford), Roger Romani (Stanford), Josh Frieman (Fermilab/U. Chicago), Jen \\ Adelman-McCarthy (Fermilab), Andrew Becker (U. Washington), Fritz DeJongh (Fermilab), Ben \\ Dilday (U. Chicago), Juan Estrada (Fermilab), John Hendry (Fermilab), Jon Holtzman (NMSU), \\ Jared Kaplan (Stanford), Rick Kessler (U. Chicago), Hubert Lampeitl (Fermilab), John Marriner \\ (Fermilab), Gajus Miknaitis (U. Washington), Douglas Tucker (Fermilab), John Barentine (APO), \\ Roger Blandford (KIPAC/Stanford), Howard Brewington (APO), Jack Dembicky (APO), Mike Harvanek \\ (APO), Suzanne Hawley (U. Washington), Craig Hogan (U. Washington), David Johnston (Princeton), \\ Steve Kahn (KIPAC/Stanford), Bill Ketzeback (APO), Scot Kleinman (APO), Jerzy Krzesinski (APO), \\ Dennis Lamenti (SFSU), Dan Long (APO), Russet McMillan (APO), Peter Newman (APO), Atsuko \\ Nitta (APO), Robert Nichol (Portsmouth), Ryan Scranton (U. Pittsburgh), Erin Sheldon (U. Chicago), \\ Stephanie Snedden (APO), Chris Stoughton (Fermilab), Don York (U. Chicago), and the SDSS \\ Collaboration
}

\begin{abstract}
In preparation for the Supernova Survey of the Sloan Digital Sky Survey (SDSS) II, a proposed 3-year extension to the SDSS, we have conducted an early engineering and science run during the fall of 2004, which consisted of approximately 20 scheduled nights of repeated imaging of half of the southern equatorial stripe. Transient supernova-like events were detected in near real-time and photometric measurements were made in the five SDSS filter bandpasses with a cadence of $\sim 2$ days. Candidate type Ia supernovae ( $\mathrm{SNe}$ ) were pre-selected based on their colors, light curve shape, and the properties of the host galaxy. Follow-up spectroscopic observations were performed with the Astrophysical Research Consortium 3.5m telescope and the $9.2 \mathrm{~m}$ Hobby-Eberly Telescope to confirm their types and measure the redshifts. The 2004 campaign resulted in 22 spectroscopically confirmed $\mathrm{SNe}$, which includes 16 type Ia, 5 type II, and 1 type Ib/c. These SN Ia will help fill in the sparsely sampled redshift interval of $z=0.05-0.35$, the so-called 'redshift desert', in the Hubble diagram. Detailed investigation of the spectral properties of these moderate-redshift SNe Ia will also provide a bridge between local SNe and high-redshift objects, and will help us understand the systematics for future cosmological applications that require high photometric precision. Finally, the large survey volume also provides the opportunity to select unusual supernovae for spectroscopic study that are poorly sampled in other surveys. We report on some of the early results from this program and discuss potential future applications.
\end{abstract}

\section{Introduction}

One of the three primary scientific components of SDSS II, a proposed three-year extension to the SDSS, is a time domain study, which involves repeated imaging of the southern equatorial stripe for three threemonth periods during the Fall months of $2005-2007$. The Supernova Survey is a major subcomponent of this study, and its primary goal is to obtain highquality, multi-color light curves of $\sim 200 \mathrm{SN}$ Ia in the intermediate redshift interval of $z=0.05-0.35$. This complements existing searches for SNe at low redshifts (e.g., Lick Observatory Supernova Search, Carnegie Supernova Program, Nearby Supernova Factory) and those at high redshifts (e.g., CFHT Supernova Legacy Survey, ESSENCE), and will not be targeted by other telescopes in the coming years. The SDSS $2.5 \mathrm{~m}$ telescope is particularly well-suited for this type of search with its ability to survey large areas of the sky to moderate depths in several filters all in a single night of observing. In addition, it allows us to take advantage of one of the most well-calibrated photometric systems that is currently available, which is crucial to help understand and quantify the systematic uncertainties associated with SNe Ia as cosmological distance indicators.

In preparation for SDSS II, we have conducted a
Table I List of SDSS $2.5 \mathrm{~m}$ imaging scans of strip $82 \mathrm{~N}$ for the Fall 2004 campaign

\begin{tabular}{ccccc}
\hline Date & run & \multicolumn{3}{c}{ RA Range (hrs) } \\
\hline $9 / 22$ & 4894 & 19.62 & - & 4.90 \\
$9 / 24$ & 4895 & 20.69 & - & 3.98 \\
$10 / 8$ & 4896 & 22.08 & - & 4.14 \\
$10 / 10$ & 4874 & 19.95 & - & 5.82 \\
$10 / 16$ & $4894 / 5$ & 21.88 & - & 4.64 \\
$10 / 18$ & 4899 & 0.66 & - & 4.14 \\
$10 / 20$ & 4805 & 0.14 & - & 4.73 \\
$10 / 24$ & 4917 & 19.74 & - & 3.47 \\
$11 / 3$ & 4927 & 20.64 & - & 4.12 \\
$11 / 5$ & 4933 & 20.53 & - & 4.16 \\
$11 / 10$ & 4948 & 1.27 & - & 4.11 \\
\hline
\end{tabular}

pilot study during the Fall of 2004 using the SDSS $2.5 \mathrm{~m}$ imaging telescope. This run consisted of repeated scans of half of the southern equatorial stripe over a period of $\sim 1.5$ months scheduled with a cadence of approximately 2 days. The area covered is approximately half of that planned for SDSS II and the duration is $\sim 6$ times shorter than the total duration over three years, which makes this pilot run 

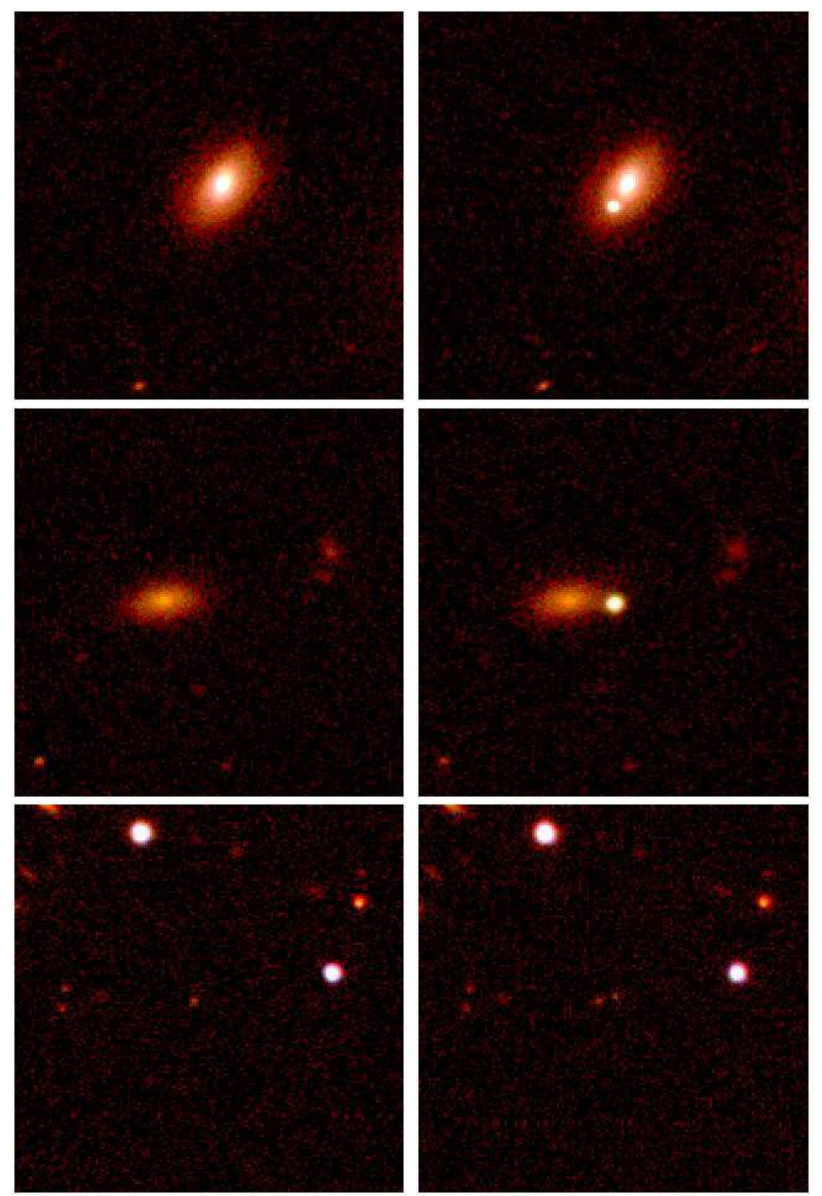

Figure 1: SDSS images of three type Ia SNe discovered and spectroscopically confirmed during the 2004 campaign - from top to bottom, SN2004hu $(z=0.0487)$, SN2004ie $(z=0.0513)$, and SN2004if $(z=0.322)$. The left panels show the template images before the supernova, which are shown on the right panels. The images are $1^{\prime} \times 1^{\prime}$ and are all centered on the locations of the supernovae.

$\sim 1 / 12$ of the size of SDSS II. We report here some of the early results from this program, which resulted in 22 spectroscopically identified SNe and light curves of an additional $\sim 2$ dozen unconfirmed events. We also briefly discuss our plans for SDSS II. Although the primary goal of the Supernova Survey is to study the properties of intermediate-redshift type Ia SNe, its large survey volume will enable us to find a large number of other types of SNe including peculiar ones as well. We briefly comment on a few potential applications.

\section{Observations}

\subsection{SDSS Imaging and Target Selection}

SDSS imaging observations of half of the southern
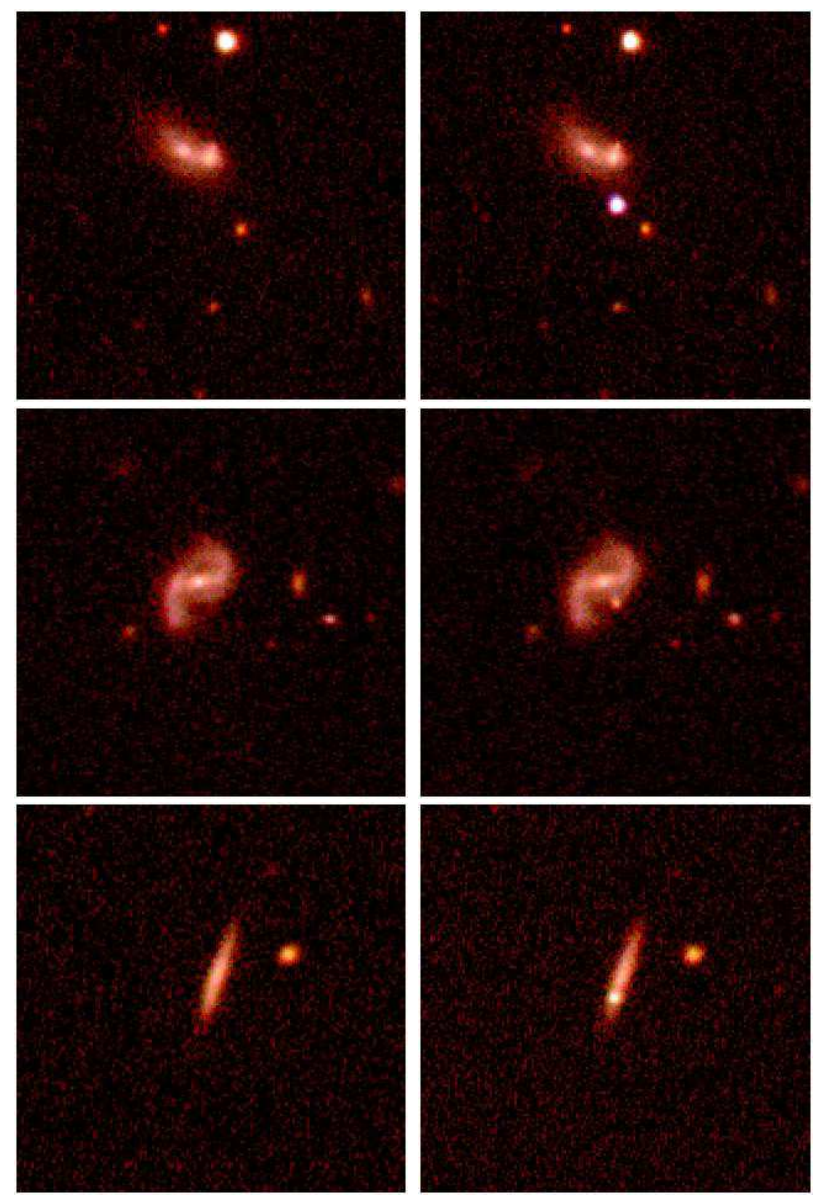

Figure 2: Same as in Figure 1 for, from top to bottom, SN2004hx (type II; $z=0.0143)$ ), SN2004ht (type II; $z=0.0668$ ), and SN2004ib (type $\mathrm{Ib} / \mathrm{c} ; z=0.0565$ ).

equatorial stripe ( $\operatorname{strip} 82 \mathrm{~N}$ ) were planned for approximately 20 nights between Sept. 21 and Nov. 15, 2004. This region covers approximately 150 square degrees of the sky, and we were able to obtain nearly complete scans of the strip on 6 nights and partial scans during another 5 nights (Table I). Note the presence of relatively long gaps during $9 / 24-10 / 8$ and 10/24 $11 / 3$, which were mostly bright time. The ranges in RA shown are only approximate.

Processed ugriz images were generated in near realtime at Apache Point Observatory (APO) and the $g$ and $r$ frames were run through a pipeline software that searches for transient events through image subtraction. During this run, we considered sources that were detected with a statistical significance of at least $\sim 10 \sigma$ in the subtracted images of both the $g$ and $r$ bands for manual inspection. This relatively high threshold naturally biased our selection towards lower redshift SNe and to those that are well-separated from the cores of bright host galaxies. An additional requirement of positional matches in the $g$ and $r$ images, whose exposures are separated by $\sim 5$ minutes, removed most fast-moving asteroids. Matches with pre- 
viously catalogued variable objects (stars and AGN) and with bright stars were vetoed in the software as well. A few SNe that are easily visible in the raw images are shown in Figures 1 and 2

Objects that made it through the automated selection were then inspected one by one and prioritized according to their likeliness to be SNe. This was done after every imaging run with the SDSS $2.5 \mathrm{~m}$. Since only the $g$ and $r$ bands were processed automatically though image subtraction, we did not make full use of the color information for initial photometric typing of the events. In general, objects that appear in the differenced image near the core of the host galaxy are ranked low, since they are relatively more likely to be AGNs. Unresolved point-like sources that are isolated from any potential host galaxy are likely to be variable stars or QSOs, and were also given low priority. All of the remaining events are potential SNe. For SDSS II, all images will be processed automatically through image subtraction in $g, r$, and $i$. In addition, resulting frames containing potentially interesting objects will be rapidly processed through image subtraction in $u$ and $z$ as well, allowing us to make use of the full color information for photometric typing of candidate SNe.

Table II List of Spectroscopically Confirmed SNe from the Fall 2004 Campaign [1]

\begin{tabular}{|c|c|c|c|c|}
\hline IAUC ID & SDSS ID & Type & Redshift & Telescope \\
\hline $2004 \mathrm{ht}$ & SN24 & II & 0.0668 & ARC/HET \\
\hline 2004hu & SN15 & Ia & 0.0487 & $\mathrm{ARC}$ \\
\hline $2004 \mathrm{hv}$ & SN9 & II & 0.0613 & $\mathrm{ARC}$ \\
\hline $2004 \mathrm{hw}$ & SN10 & Ia & 0.0601 & HET \\
\hline 2004hx & SN18 & II & 0.0143 & $\mathrm{ARC}$ \\
\hline 2004hy & SN12 & II & 0.0587 & $\mathrm{ARC}$ \\
\hline $2004 \mathrm{hz}$ & SN5 & Ia & 0.1427 & HET \\
\hline 2004ia & SN30 & Ia & 0.1431 & $\mathrm{ARC}$ \\
\hline 2004ib & $\mathrm{SN} 20$ & $\mathrm{Ib} / \mathrm{c}$ & 0.0565 & HET \\
\hline $2004 \mathrm{ic}$ & SN111 & II & 0.093 & $\mathrm{ARC}$ \\
\hline 2004id & SN19 & Ia & 0.1444 & $\mathrm{ARC}$ \\
\hline 2004ie & SN83 & Ia & 0.0513 & $\mathrm{ARC}$ \\
\hline 2004if & SN25 & Ia & 0.322 & HET \\
\hline 2004ig & SN133 & Ia & 0.1831 & $\mathrm{ARC}$ \\
\hline 2004ih & SN128 & Ia & 0.1538 & $\mathrm{ARC}$ \\
\hline 2004ii & SN172 & Ia & 0.1973 & $\mathrm{ARC}$ \\
\hline $2004 \mathrm{ij}$ & SN247 & Ia & 0.218 & HET \\
\hline $2004 \mathrm{ik}$ & SN242 & Ia & 0.1691 & HET \\
\hline 2004il & SN191 & Ia & 0.108 & $\mathrm{ARC}$ \\
\hline 2004im & SN176 & Ia & 0.134 & ARC \\
\hline 2004in & SN194 & Ia & 0.1612 & $\mathrm{ARC}$ \\
\hline 2004io & SN171 & Ia & 0.1502 & $\mathrm{ARC}$ \\
\hline
\end{tabular}

\subsection{Additional Imaging Observations}

For a handful of the spectroscopically confirmed $\mathrm{SNe}$, additional imaging observations were performed with the New Mexico State University (NMSU) 1m and the Astrophysical Research Consortium (ARC) $3.5 \mathrm{~m}$ telescopes at APO. The images were taken with the SDSS $g, r$, and $i$ filters whenever possible. The main objectives of these observations were to obtain more densely-sampled light curves on a handful of candidates and to provide additional photometric points beyond the survey duration especially for those events that were discovered towards the end of the run. This was especially important for the test run, since its 1.5month duration was not optimal for harvesting full SNe light curves. Since all of these telescopes are at APO, however, they could not be used as replacements to fill in light curve points during poor weather conditions.

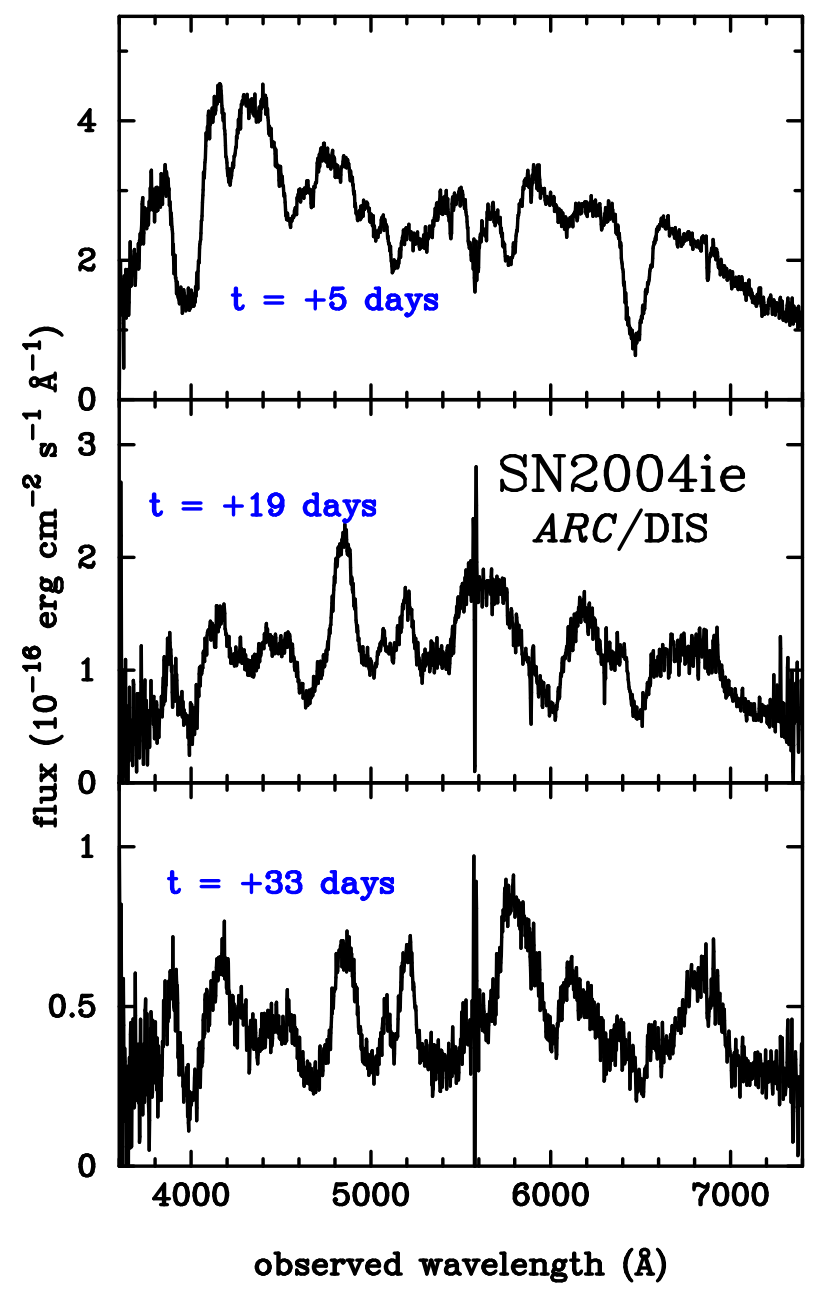

Figure 3: ARC spectra of SN2004ie + galaxy $(z=0.0513)$ at $t=+5$ days (top),+19 days (middle), and +23 days (bottom) after maximum. The noisy region near $\lambda=5600 \AA$ is due to the dichroic cutoff of the red and blue cameras. 


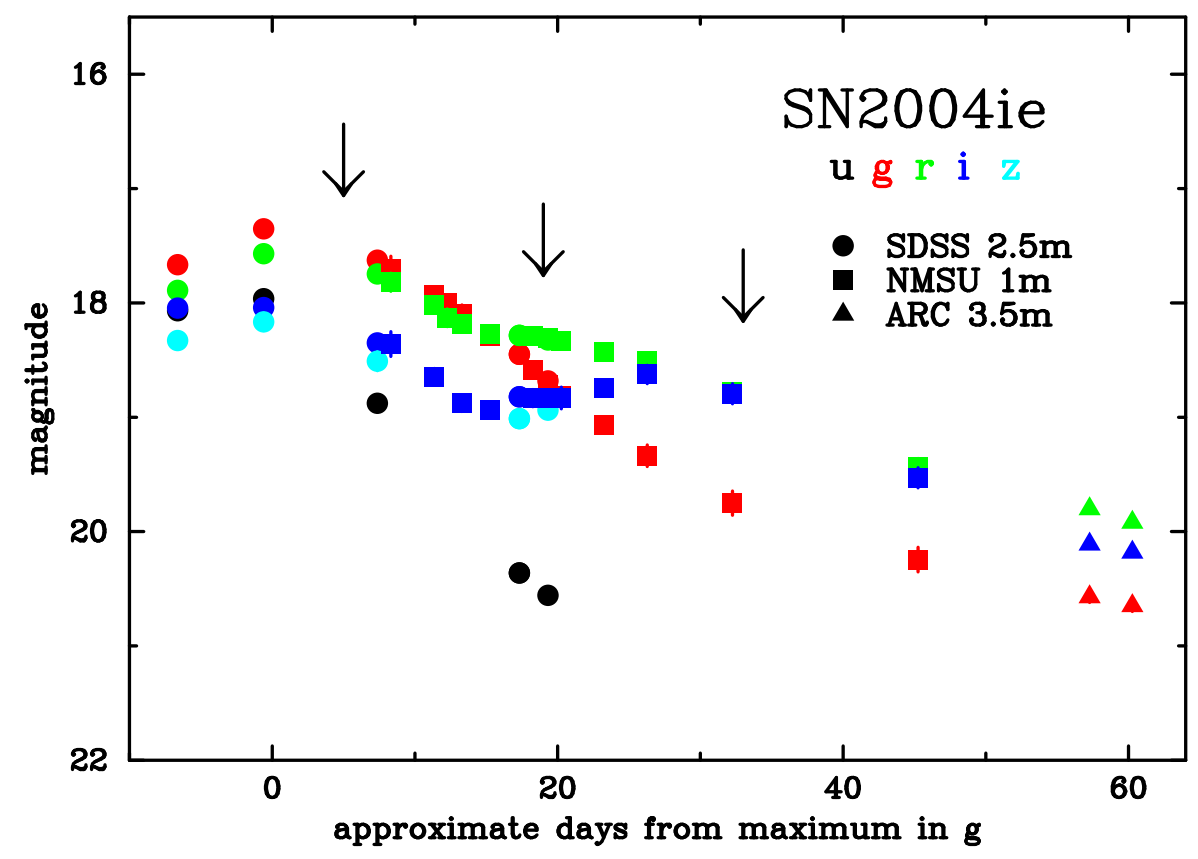

Figure 4: Preliminary multi-color light curves of the type Ia SN2004ie $(z=0.0513)$. Measurements from the SDSS $2.5 \mathrm{~m}$, NMSU $1 \mathrm{~m}$, and the ARC $3.5 \mathrm{~m}$ are denoted as filled circles, squares, and triangles, respectively. The three arrows indicate epochs of spectroscopy with the ARC $3.5 \mathrm{~m} / \mathrm{DIS}$.

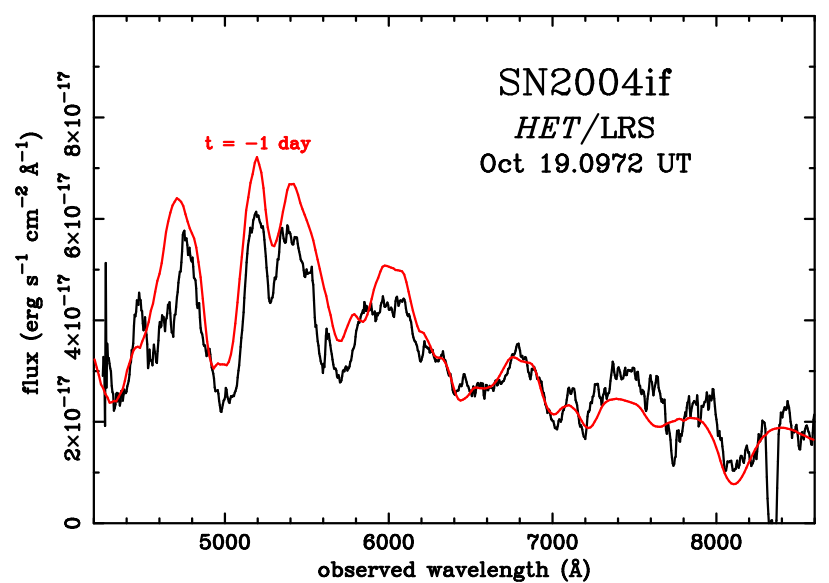

Figure 5: Observed spectrum of the type Ia SN2004if $(z=0.322)$ obtained with the HET (black) and the best-matching template spectrum (red).

\subsection{Follow-up Spectroscopy}

Spectroscopic observations were planned with Double Imaging Spectrograph (DIS) on the ARC $3.5 \mathrm{~m}$ telescope at APO and the Low Resolution Spectrograph (LRS) on the queue-scheduled 9.2m HobbyEberly Telescope (HET) at McDonald Observatory. In most cases, relatively bright events with $g \lesssim 20.6 \mathrm{mag}$ were targeted with the ARC $3.5 \mathrm{~m}$ and fainter sources were put in the HET queue usually at high priority. There were a few exceptions, however, due to scheduling constraints and availability of telescope time on

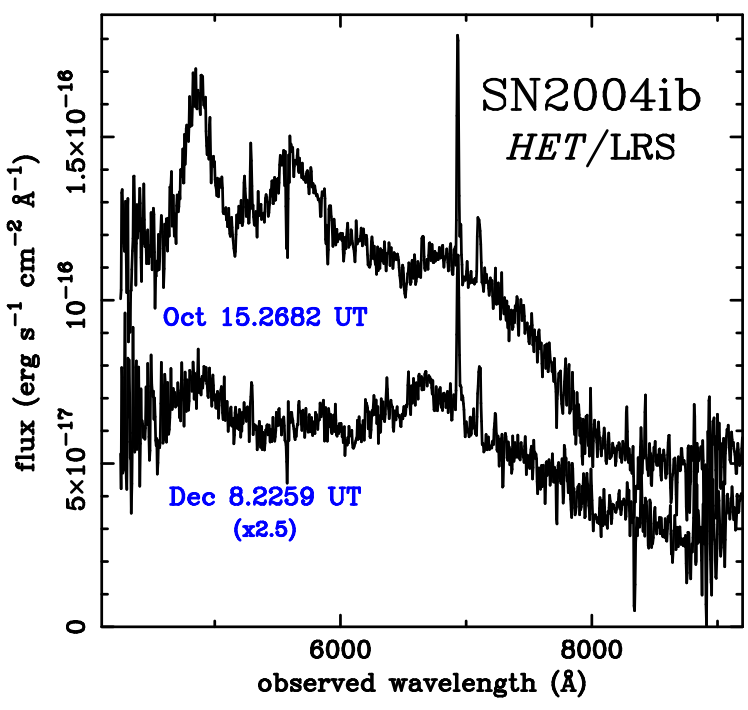

Figure 6: Multi-epoch HET spectra of the type Ib/c hypernova SN2004ib + galaxy $(z=0.0565)$.

any given night. Both observatories are again generally affected by the same weather pattern.

Spectral classification of type Ia SNe is least ambiguous with spectra obtained near maximum light [2], so every attempt was made to perform the observation within $\sim 2$ weeks of discovery. This did not always work in practice, however, particularly for the queue-scheduled HET, which is affected by conflicts with other competing programs, in addition to the 


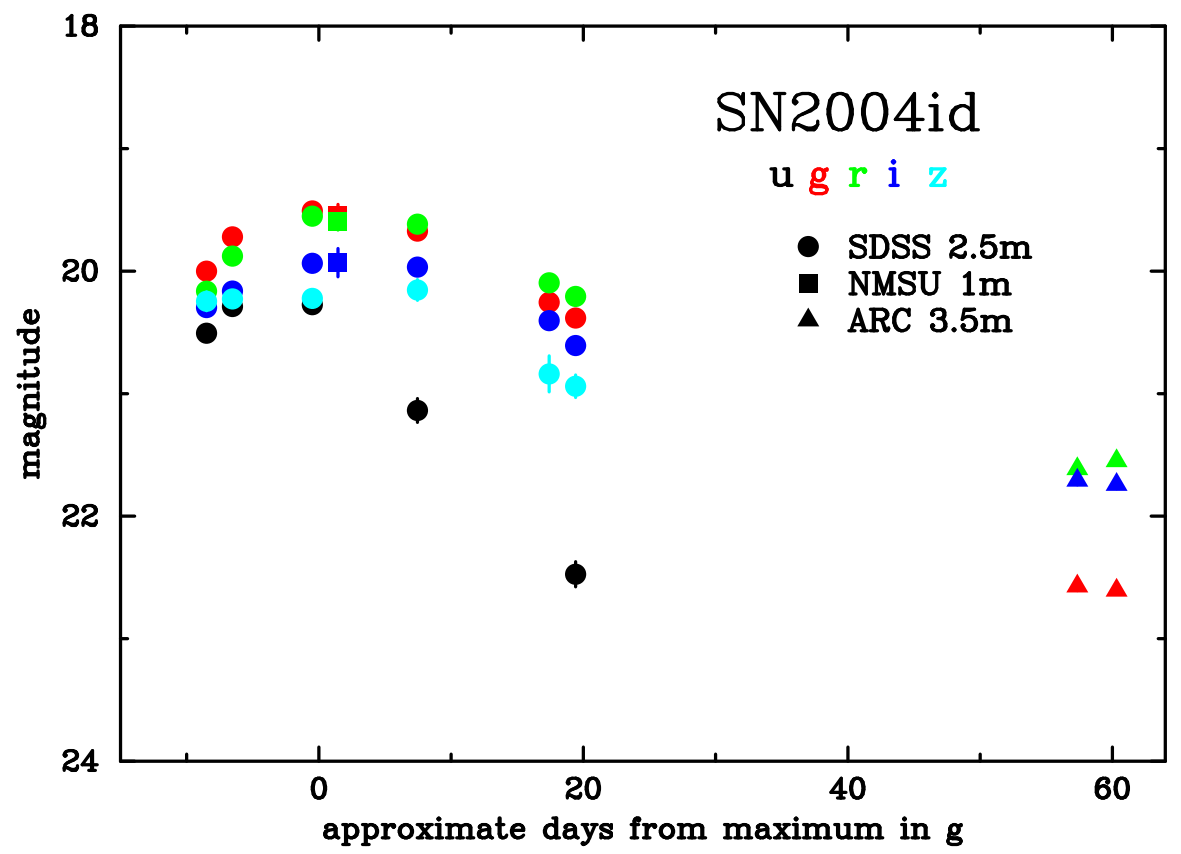

Figure 7: Same as in Figure 4 for the type Ia SN2004id + galaxy $(z=0.1444)$.

usual weather and moon constraints.

The redshifts were typically inferred by taking the total measured spectrum (SN + galaxy) and crosscorrelating against a number of template galaxy spectra using the IRAF package RVSAO [3]. When the supernova was well-separated from the host and no obvious galaxy light was detected, the spectra were cross-correlated with template SN spectra. The redshifts measured from the host galaxy spectra are generally more accurate than redshifts derived from broad features of the SN spectrum. In all cases, however, the two methods agreed to within their statistical errors.

In the Fall 2004 campaign, we were able to spectroscopically confirm 16 type Ia, 6 type II, and 1 type $\mathrm{Ib} / \mathrm{c}$, which are listed in Table $\Pi$ along with their redshifts. These include only sources from which we were able to obtain a SN spectrum. There are a handful of additional sources for which the host galaxy redshift is measured, and the SN light curve is consistent with that of a Ia at this redshift. These are considered as likely type Ia SNe, and are not included in Table

\section{Light Curves}

Photometric measurements were performed by first registering the template and search images using point sources near the SN. PSF magnitudes of the point sources in the search frame were measured and compared to the catalog PSF magnitudes released in DR3 [4] to determine the zeropoints in each of the five colors. The images were then cross-convolved (template frame convolved with search PSF; search frame con- volved with template PSF) to minimize systematics. PSF magnitudes were finally measured on the subtracted images. Multi-color light curves of two of the best observed SN Ia are shown in Figures 4 and 7

Detailed light curve fits are in progress, but a preliminary analysis indicates that SN2004ie is a normal type Ia with $\Delta m_{15}(B)=1.0$ with very little host galaxy extinction, consistent with the image shown in the middle panel of Figure 1 which shows the SN near the edge of its host galaxy.

\section{The Supernova Survey of SDSS II}

The Supernova Survey of SDSS II will observe the entire equatorial stripe 82 , which covers the coordinate ranges of $-50^{\circ}<\alpha<+60^{\circ}$ and $-1.25^{\circ}<\delta<$ $+1.25^{\circ}$, with a cadence of 2 days from the beginning of September to the end of November for the next three years. This is approximately 4 times the size of the Fall 2004 campaign per year. With the discovery and confirmation of 16 type Ia SNe, we can estimate that at least $\sim 16 \times 4=64$ SNe Ia will be discovered per year with SDSS II. We expect this to be a lower limit, since (1) during the test run, our thresholds were not optimized to search for faint events in the redshift range of $0.2 \lesssim z \lesssim 0.35$, which spans a much larger volume, (2) extending the survey duration from 1.5 months to 3 months results in more than a factor of two increase in the number of events with well-sampled light curves, and (3) the spectroscopic follow-up plans for SDSS II are also more extensive. We expect to request approximately 33 half-nights for 
spectroscopic follow-up from the ARC $3.5 \mathrm{~m}$ this Fall. The HET will devote at least 50 hours of observing time primarily for the $z \gtrsim 0.2 \mathrm{Ia}$, and Ohio State has committed 30 nights per year of MDM $2.4 \mathrm{~m}$ time for this project. In addition, proposals are being submitted to ESO, Subaru, and the William Herschel Telescope on La Palma.

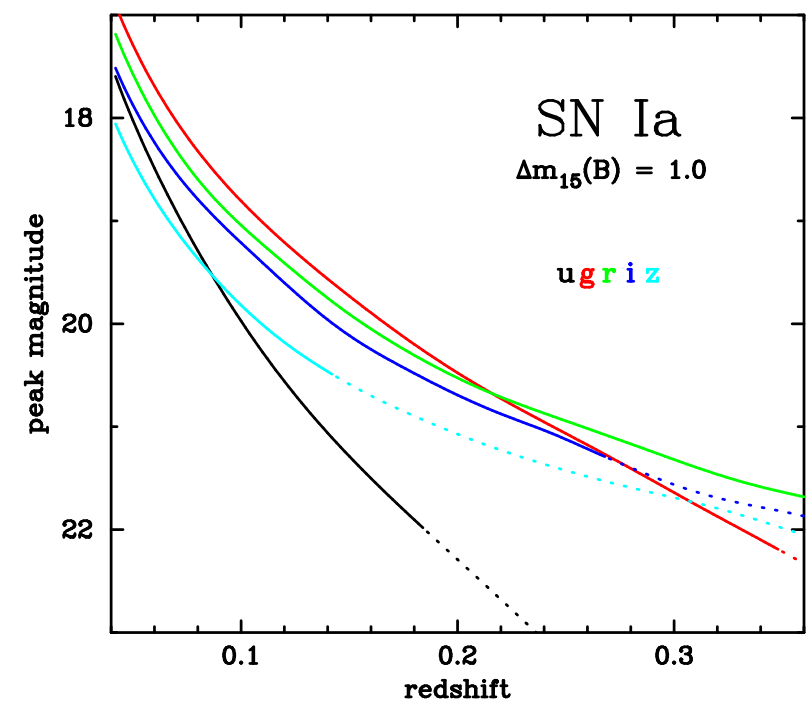

Figure 8: Peak magnitudes of a normal type Ia SN with $\Delta m_{15}(B)=1.0$ [5] as a function of redshift calculated using a set of template spectra provided by A. Riess (2004, private communication) in each of the SDSS filters. Points on each of the curves that fall below the $95 \%$ point-source detection repeatability values are shown by dotted lines.

Figure 8] shows the expected peak ugriz magnitudes for a normal type Ia with $\Delta m_{15}(B)=1.0$, where $\Delta m_{15}(B)$ is the increase in $B$-band magnitude 15 days after peak [5]. For the nearby events $(z \lesssim 0.15)$, useful light curves will be acquired in all five colors, giving better constraints on the reddening. For more distant events $(z \gtrsim 0.2)$, the $u$ and $z$ magnitudes will likely fall below the detection limit.

We plan to also run the $i$-band frames through realtime image subtraction in addition to the $g$ and $r$ frames, and use an improved version of the image subtraction pipeline for automated candidate selection. We expect these changes to result in an increase in the detection efficiency of SNe Ia near the high redshift end of $z \sim 0.3$ without dramatically increasing the number of candidates for manual inspection. We also plan to manually perform photometric measurements on the $u$ and $z$ images of candidates that make through the cut, and use the full color information for pre-typing. Photometric redshifts of host galaxies can also be useful for this process. We continue to optimize our selection criteria using the data acquired in fall 2004.

We also expect to find and measure light curves of $\sim 100$ type II SNe out to a redshift of $z \sim 0.2$ and possibly higher. The large sample up to moderate depths will be extremely valuable for studying in detail the exciting possibility of using type II SNe as standardized candles and applications for cosmology 6]. Finally, we note that the large volume of this survey also provides a unique opportunity to find unusual SNe that are poorly sampled in other surveys. One such example is the class of luminous type $\mathrm{Ib} / \mathrm{c}$, some of which are thought to be associated with gamma-ray bursts. Initial color-typing and photometric redshifts of the host galaxies can pre-select sources for followup spectroscopy in the optical and possibly in other wavelengths as well.

\section{Acknowledgments}

The authors would like to thank the HET resident astronomers for their quick cooperative responses, and their continuous effort throughout this run. MS thanks Nick Suntzeff for valuable advice. Funding for the creation and distribution of the SDSS Archive has been provided by the Alfred P. Sloan Foundation, the Participating Institutions, the National Aeronautics and Space Administration, the National Science Foundation, the U.S. Department of Energy, the Japanese Monbukagakusho, and the Max Planck Society. The SDSS Web site is http://www.sdss.org/. The SDSS is managed by the Astrophysical Research Consortium (ARC) for the Participating Institutions. The Participating Institutions are The University of Chicago, Fermilab, the Institute for Advanced Study, the Japan Participation Group, The Johns Hopkins University, the Korean Scientist Group, Los Alamos National Laboratory, the Max-Planck-Institute for Astronomy (MPIA), the Max-Planck-Institute for Astrophysics (MPA), New Mexico State University, University of Pittsburgh, University of Portsmouth, Princeton University, the United States Naval Observatory, and the University of Washington.

\section{References}

[1] Adelman-McCarthy, J., Becker, A., Dejongh, F., Dilday, B., Estrada, J., Frieman, J., Kaplan, Kessler, R., Holtzman, J., J., Lampeitl, H., Marriner, J., Miknaitis, G., Romani, R., Sako, M., \& Tucker, D., 2005, IAUC, 8481

[2] Filippenko, A. F. 1997, ARA\&A, 35, 309

[3] Kurtz, M. J., \& Mink, D. J. 1998, PASP, 110, 934

[4] Abazajian, K., et al. 2005, AJ, 129, 1755

[5] Phillips, M. M. 1993, ApJL, 413, L105

[6] Hamuy, M, \& Pinto, P. A. 2002, ApJL, 566, L65 